\title{
LA VIE DU COUPLE À LA CINQUANTAINE TRANSFORMATIONS ET ACCIDENTS
}

\author{
Sylvain Mimoun* \\ *Centre d'Andrologie et de la Reproduction, Service d'Urologie de l'Hôpital Cochin (Pr B. Debré) \\ 27, rue du Faubourg St Jacques - 75014 PARIS
}

COUPLE LIFE AT THE FIFTIES ; CHANGES AND ACCIDENTS. After having evoked the male and female reactions towards time passing, the different crises which may surge at this time and the particularity of couple counselling at this age, the author mainly evokes the situations generating conflicts and couple crises because it is in these cases that one better perceives the acting mechanisms. Key-words : menopause, andropause, couple, sexuality. Andrologie, 1991, 1: 36-37

La vie du couple à la cinquantaine peut être sans histoires, sans vagues, sans transformations spectaculaires et sans accidents. Mais nous ne parlerons ici que des situations qui sont sources de conflits et de crises conjugales puisque c'est dans ces cas que l'on perçoit mieux les mécanismes agissants.Avant d'appréhender les cas de figure les plus fréquents qui surgissent au détour de la vie d'un couple à la cinquantaine, nous allons dire quelques mots de la différence des attitudes des hommes et des femmes face au temps qui passe et face aux crises de la vie.

\section{LES FEMMES ET LES HOMMES FACE AU TEMPS}

Les femmes ont un rapport au temps différent de celui des hommes. Par la puberté, les règles, l'accouchement, la ménopause, le temps est inscrit dans leur corps. Il est intégré intérieurement et non pas extérieurement.

Les hommes, au contraire, n'ont pas de signe physique aussi net. Ainsi le temps apparaît-il aux hommes plus impalpable, plus réversible, plus élastique... Leurs références temporelles essentielles sont extérieures à leur corps. C'est par le temps social que les hommes sont le plus souvent déterminés. Qu'il s'agisse des dates de leurs différentes activités, du service militaire, de la retraite, etc... ils sont également déterminés, bien sûr, par le temps des autres, celui de leur femme en particulier. C'est d'ailleurs souvent quand celle-ci a sa ménopause qu'ils prennent conscience de leur propre vieillissement Pour Eliane Perrin, sociologue, cela pourrait expliquer un certain nombre de comportements masculins entre quarante et cinquante ans, consistant par exemple à prendre une femme plus jeune afin de vivre lillusion de rajeunir. (4).
Par ailleurs, les hommes et les femmes ne réagissent pas du tout de la même façon face à une crise, c'est-à-dire une situation en rupture avec l'équilibre établi. En effet, quand une femme a des difficultés, elle aura tendance à s'introspecter et à rattacher ce qu'elle ressent physiquement et psychologiquement à son vécu interne. Elle est prête par exemple à rechercher de l'aide auprès des médecins et des psychothérapeutes. Les hommes, par contre, ont plutôt tendance à réagir aux situations de crise en agissant sur l'extérieur: trouver un nouvel emploi, déménager, s'engager dans un mouvement, changer de femme, etc... Ces modes de réaction différents peuvent aboutir à un véritable "dialogue de sourds" dans le couple et à une absence totale de communication.

\section{LES CRISES DE LA VIE}

Il est classique de dire que si la période de la ménopause est pour les femmes une période difficile, une période de crise, il n'en est pas de même pour les hommes à cette époque de leur vie. Or une étude menée à Genève de 1982 à 1984 par Myriam de Senarclens et Eliane Perrin (3) va à l'encontre de ces idées. Les auteurs ont analysé les périodes de vie difficile pour les hommes et pour les femmes aux différents âges. Ce qui est surprenant dans cette étude est que pour les femmes à partir de 46 ans, c'est-à-dire au moment de la péri-ménopause, les moments difficiles diminuent. Les hommes ont par contre une crise de vie très importante entre 43 et 52 ans (fin de la période d'ascension sociale et de la prise de conscience de cette fin). Les résultats de cette étude qu'il serait souhaitable de développer sur une population plus vaste, sont en contradiction avec les discours médicaux et sociologiques habituels. Ces données nous semblent intéressantes à double titre. D'une part parce qu'elles laissent à penser que les femmes peuvent mieux traverser des difficultés de vie à la période ménopausique, (comme chacun sait l'attente d'un mal-être l'induit plus ou moins) d'autre part parce que cela peut réconforter certains hommes qui, à 45 ou 50 ans, ont l'impression de traverser "tout seul", un moment difficile.

En effet, autant les femmes sont surpréparées à aborder la ménopause, tant cette période de crise est reconnue socialement, autant rien n'est reconnu ni admis pour les hommes. Ce qui les rend très démunis et les oblige à faire face isolément à des difficultés considérées comme personnelles et anormales. Les hommes vivent leur cinquantaine d'autant plus mal qu'ils pensent devoir être au meilleur de leur forme.

Si l'homme est au chômage, ou en pré retraite, quand sa femme aborde la ménopause, le climatère est souvent tenu pour responsable des conflits conjugaux. Pourtant, avec le chômage, le mari passe son temps à la maison, ce qui ennuie incontestablement la femme qui se sent "envahie" et gênée dans la gestion de son temps domestique. Le mari, quant à lui, se sent souvent dépouillé de son prestige professionnel et inadapté par rapport à son temps nouvellement disponible. Et cela est d'autant plus marqué si la femme continue à travailler.

\section{LES DIFFICULTES SEXUELLES}

Les troubles sexuels sont fréquents autour de la cinquantaine. Il n'est pas surprenant que dans ces moments de tension interne, dans cette traversée d'une période de crise, des hommes aient des pensées parasites même au moment des rapports sexuels, et que cela entraîne une diminution de l'érection. Cette baisse, même légère, suffit à les inquiéter notablement au point de les faire entrer dans un véritable cercle infernal où la peur de l'échec entraîne l'échec et ainsi de suite... La femme, elle, ressent des malaises (bouffées de chaleur, palpitations, insomnies...) qui lui donnent un sentiment d'étrangeté. Sur le plan sexuel, il est fréquent de rencontrer, dès l'installation de la ménopause, une baisse de la libido et une difficulté à avoir un orgasme, les dyspareunies dues à la carence cestrogénique survenant en général plus tard. La plupart du temps, les femmes qui se plaignent de la perte subite du désir sexuel sont celles qui avaient une sexualité harmonieuse et satisfaisante auparavant. La difficulté, nouvelle, à avoir un orgasme est sans doute liée au ralentissement des réactions sexuelles de la femme ménopausée. Ce ralentissement sera provisoire si la femme et le couple arrivent à s'adapter à ces nouvelles données. Une plus longue "préparation" est nécessaire mais les réactions sexuelles restent satisfaisantes. Par contre si le couple, surpris par le manque de réaction au bout du temps habituel considère que 
"quelque chose est cassé" et qu'il n'y a rien à faire (puisque cela est dû la ménopause) le trouble s'installe et auto-entretient le cercle vicieux d'échec. Il est vrai que les ajustements nécessaires sont rarement faits. Le partenaire vieillissant craint d'avoir lui aussi des problèmes, voire même se remet en question du fait de l'absence de réaction de sa femme.

Bien souvent, la peur de l'échec conduit l'homme à abréger les préliminaires amoureux et accroit ainsi la mésentente sexuelle. Tout ceci est encore plus vrai si la femme n'était pas satisfaite ou si elle était peu intéressée par la sexualité. La ménopause devient alors l'alibi pour cesser toute activité sexuelle. De plus dans les vieux couples où la "routine" s'est installée, la femme à cette époque de sa vie doute de son charme et de sa féminité. Elle se sent d'autant moins désirable que son partenaire n'exprime plus, ou n'ose plus exprimer le désir qu'il a encore pour elle. Quelquefois les bouffées de chaleur peuvent être l'objet d'affectueuses plaisanteries ou entrainer "la chambre à part".

D'autre part, la période de la cinquantaine est celle où les deux conjoints vont se retrouver "volontiers" face à face, que les enfants soient présents mais adultes, ou partis. Les couples les plus menacés à cet égard, sont ceux dont le mariage a été suivi très rapidement d'une grossesse. Ils n'ont jamais été seuls et il est fréquent qu'une fois les enfants partis, ils ne sachent plus quoi se dire. Leurs problèmes relationnels ont plus d'accuité qu'auparavant. Quand la ménopause s'installe dans un couple "désertique", c'est un prétexte de plus pour dire adieu à la sexualité. Quand elle fait irruption dans un climat déjà conflictuel, on observe alors des phénomènes exacerbés, des troubles fonctionnels bruyants chez la femme comme chez l'homme. Ces symptômes peuvent être plus ou moins consciemment utilisés dans l'enjeu d'un conflit.

D'autres fois le départ des enfants est souhaité "pour se retrouver" et le "nid" est considéré comme trop plein. C"est le cas, lorsque pour des raisons dues aux études, ou aux conditions matérielles, la maison est pleine des enfants et de leurs problèmes. "On voudrait souffler, pas un dimanche tranquille! Cela majore les tensions avec mon mari" affirme Mme D. qui consulte pour mésentente sexuelle.

Quelquefois enfin l'arrivée des petits-enfants peut être l'objet de découvertes que l'on n'a pas eu la possibilité de faire avec ses enfants. Ce peut être l'occasion d'une nouvelle complicité dans le couple. Résumons-nous, habituellement on rencontre plusieurs cas de figure.

\section{1 - La cinquantaine à problèmes :}

Souvent dans ces cas, la cinquantaine est considérée comme une période où il n'y a plus rien à attendre. Il s'agit souvent de femmes (ou d'hommes), qui ont été, leur vie durant, déçus dans leur attente affective. Pour eux, l'origine du malaise est toujours lié à l'entourage, à l'extérieur. Ils se plaignent en permanence du conjoint des enfants, du gendre, de la belle-fille... L'abord des difficultés actuelles peut être efficient si la rancœur n'est pas trop profondément enracinée chez les deux partenaires.

\section{2-Les réactions dépressives:}

La femme reste fixée au moment de la perte symbolique de sa féminité. "Tout a commencé au moment de ma ménopause". L'homme lui reste fixé au traumatisme social (perte du travail, préretraite...) Il faut souligner combien cette dépression, contemporaine de ces événements peut être dissimulée sous des plaintes physiques (fatigue, douleurs...). Quelquefois l'homme craque parce qu'à tort ou à raison il se sent remis en question. Par exemple quand il découvre que sa femme a un amant. Indépendamment de la peine et de la jalousie quilil peut ressentir, ce qui peut le désarçonner c'est la mise en compétition avec le rival et surtout le sentiment avoué ou non, de ne plus être à la hauteur.

\section{3 - Les bons cas :}

C'est-à-dire quand chez la femme et chez l'homme, il y a très peu de perturbations physiques et psychiques. Souvent dans ces cas l'érotisme au sens large, ne se laisse pas abattre. Les défaillances, si elles existent, sont passagères. Cette catégorie semble concerner près de $60 \%$ des couples. Dans ce cas, le désir est entretenu par le jeu de la séduction, par la relation de confiance, la complicité et le don de soi. Dans ces cas, les couples privilégient la complicité et le "contact" intime au détriment de l'aspect quantitatif.

\section{LA PRISE EN CHARGE THERAPEUTIQUE}

Il n'est pas rare, de nos jours, que des femmes, des hommes, ou des couples, osent consulter quel que soit leur âge pour mésentente sexuelle ou conjugale.

\section{Que peut faire le médecin dans ces cas?}

Tout d'abord, il n'aura pas la même position s'il veut se limiter à écouter, dédramatiser, informer, ou s'il se sent prêt à la prise en charge thérapeutique de ce couple. $\mathrm{Si}$, dans le premier cas, un certain nombre de mythes et de fausses croyances peuvent être ajustés, dans le deuxième, la pratique des thérapies de couples et/ou des thérapies sexuelles est indispensable même si elle n'est pas toujours suffisante. La problématique conjugale n'est pas du tout la même quand un couple a 25 ans et quil démarre sa vie commune ou s'il en a 50 et que cela fait plus de 20 ans que les conflits existent.

Un couple d'âge mûr ne repart jamais à zéro.
Les années ne peuvent être annihilées. Lors d'un remariage par exemple, l'intensité de l'investissement affectif, de la recherche d'une nouvelle forme d'existence en commun n'est plus aussi marquée que la première fois. Le mariage précédent, malgré ses imperfections, constitue une tranche de vie qui marque la personnalité.

Sur le plan plus strictement sexuel, rappelons que depuis MASTERS \& JOHNSON de nombreux protocoles thérapeutiques ont été mis au point par divers auteurs en vue du traitement des dysfonctions sexuelles. Le fait que le couple ait la cinquantaine ne change pas radicalement ces programmes, à ces différences près que les manifestations physiques de la femme tout comme celles de l'homme de 50 ans doivent être atténuées par les traitements médicaux appropriés.

Notons à ce sujet quili est très souvent proposé à l'homme de 50 ans d'avoir des injections intracaverneuses comme remède essentiel de ses troubles éventuels de l'érection. Certes l'homme a besoin d'abord et avant tout d'être rassuré sur sa propre puissance et les injections peuvent éventuellement le rassurer momentanément. Mais ceci aura peu d'effet durable si la femme n'est pas complice, si elle se sent "utilisée", si le climat conjugal se détériore etc... Les deux partenaires ont intérêt à prendre conscience que pour l'homme l'environnement et le "décor" érotique comptent beaucoup, alors que pour la femme la sexualité est plus temporelle. Elle aime l'attente, le délai, la durée...

En conclusion, disons que si un homme, une femme, ou un couple consultent, il est du rôle du médecin de leur permettre de reconnaître la différence de l'autre, et même de l'utiliser pour la satisfaction des deux. Le médecin a une position de tiers devant qui les deux partenaires peuvent parfois communiquer plus facilement. C'est ce qui peut permettre à la "crise" conjugale, non seulement d'être résolue, mais aussi d'être un nouveau départ même à la cinquantaine.

\section{REFERENCES}

Burgat J.M. La Ménopause et le Praticien. Méd. \& Hygiène, 1987, 45 : 1141-1144

Mimoun S. Gynécologie Psychosomatique. Encyc. Méd. Chir., Gynécologie 61985, A 10.11 : p.167

Perrin E., de Senarclens M., Perspectives sociopsychosomatiques : femmes et ménopause, homme et crise de la cinquantaine. Méd. Psychosom. 1988, $16: 2$

de Senarclens M. Perrin E. La ménopause et ses ambiguités. Colloque sur climatère et ménopause. Faculté de Médecine de Lisbonne - 1982

Willi J. : Crise conjugales et possibilités thérapeutiques. Méd. Hyg., 1987, 45 : 1072-1074 